\title{
Interleukin-6 as a potential molecular target in esophageal squamous cell carcinoma
}

\author{
ZHI-FEI ZHAO, JIAN-XIONG LI, RUI YE, XUAN WU, LING-LING GAO and BAO-LONG NIU \\ Department of Radiotherapy, General Hospital of PLA, Beijing 100853, P.R. China
}

Received September 1, 2014; Accepted June 2, 2015

DOI: $10.3892 / 01.2015 .3990$

\begin{abstract}
Knowledge of potential tumor markers may improve chemotherapeutic efficacy. Interleukin-6 (IL-6) expression in local tumor tissues is associated with cancer progression and poor prognosis in variety of cancer types. The aim of the present study was to investigate the role and potential application of IL-6 in determining the prognosis of esophageal carcinoma. KYSE170 and TE13 esophageal cancer cell lines were used to conduct cell- and animal-based experiments investigating biological changes and tumor behavior. Immunohistochemical analysis revealed that 70-80\% of cancer cells exhibited positive staining for IL-6, compared with $<15 \%$ of non-malignant epithelial cells. These immunohistochemical results were consistent with the mRNA expression levels detetced. The IL-6 silencing vector significantly reduced invasion and proliferation of the two cell lines and attenuated tumor growth in xenograft mouse models $(\mathrm{P}<0.05)$. The IL-6 silencing vector markedly reduced the presence of $\mathrm{Ki}-67$ (a typical proliferation marker) and microvessel density, indicating that downregulation of IL-6 levels may greatly affect tumor growth and inhibition. The IL-6 silencing vector increased E-cadherin and matrix metalloproteinase (MMP)-9 expression levels in the two esophageal carcinoma cell lines. This vector also regulated the release of IL-6 in cell supernatant and serum in KYSE170- and TE13-tumor-bearing mice. The secretion of vascular endothelial growth factor and cluster of differentiation 31 (a nuclear protein) immunoreactive molecules were also reduced by the IL- 6 silencing vector. Therefore, IL- 6 may be an important trigger in the progression of angiogenesis and endothelial tube formation within the tumor, and targeting IL-6 may be a promising strategy for the treatment of esophageal cancer.
\end{abstract}

Correspondence to: Dr Jian-Xiong Li, Department of Radiotherapy, General Hospital of PLA, 28 Fuxing Road, Beijing 100853, P.R. China E-mail: jianxiongli2003@gmail.com

Key words: cell proliferation, esophageal cancer, interleukin-6, angiogenesis

\section{Introduction}

Esophageal cancer is a gastrointestinal cancer and one of the most fatal types of cancer worldwide, accounting for $>300,000$ mortalities annually, with a high prevalence in East Asian countries, including China $(1,2)$. Despite advancements in technologies for the treatment of this cancer, the prognosis of esophageal cancer remains poor, with an extremely low overall 5-year survival rate ( 20\%) for esophageal squamous cell carcinoma (SCC) and a high mortality rate $(3,4)$. Current treatment strategies include chemotherapy, radiation therapy and surgical removal of tumors; however, none of these strategies can improve the tumor progression profile in esophageal cancer. Therefore, identification of potential molecular markers that can effectively predict the response to treatment and the prognosis of esophageal cancer has attracted the attention of researchers (5-7).

Generally, cancer is accompanied by a range of inflammatory reactions, which have been demonstrated to promote tumor proliferation, invasion and angiogenesis (8-10). Various cytokines, including interleukin (IL)-6, IL-8 and tumor necrosis factor (TNF)- $\alpha$, have been identified to be critical in the pathogenesis of numerous cancer types (11). Specifically, the proinflammatory cytokine, IL-6, is one of the most dysregulated cytokines in cancer, with its overexpression detected in the majority of tumors $(12,13)$. It has been reported that IL-6 is activated in a variety of cancer types, including breast, ovary and prostate cancer, and renal cell carcinoma, along multiple myelomas, leukemias and lymphomas. In addition, IL-6 plays a vital role in diverse physiological processes, including cell proliferation, migration, invasion, apoptosis, angiogenesis, growth and differentiation of cancer cells (14-16). Specifically, IL-6 may act to inhibit cell apoptosis and accelerate angiogenesis (in cancer), immune cell infiltration and stromal reaction, thereby aiding in the proliferation and differentiation of the tumor cells. By activating the JAK/STAT3 and PI3K/AKT signaling pathways, IL- 6 may regulate the growth and spread of cancer in the body $(12,14)$. In particular, IL-6 serum levels have been reported to be elevated in patients with esophageal carcinoma and were found to be associated with disease progression and prognosis (17). Although emerging evidence suggests a crucial role of IL-6 in a number of malignancies, its role in esophageal carcinoma remains unclear.

The present study investigated the underlying mechanisms of IL-6 in esophageal cancer. The aim was to elucidate the 
functional mechanism and role of IL-6 downregulation in esophageal cancer, as well as the association of IL-6 expression with tumor growth. The esophageal cancer cell lines, KYSE170 and TE13, were selected for the in vitro and in vivo experiments. Various biological characterization techniques, including cell proliferation, cell invasion/migration, immunoblot, immunohistochemical and immunohistofluorescence analyses, were conducted.

\section{Materials and methods}

Cell culture and reagents. The human esophageal SCC cell lines, KYSE170 and TE13, were obtained from the MD Anderson Cancer Center (University of Texas, Houston, TX, USA). The IL-6-green fluorescent protein (GFP) silencing vector [human IL-6 small hairpin RNA (shRNA) constructs in retroviral GFP vector] and GFP-control vector (non-effective scrambled shRNA cassette in retroviral GFP vector) were purchased from Origene Technologies, Inc. (Rockville, MD, USA). Cells were cultured in Dulbecco's modified Eagle's medium supplemented with $10 \%$ fetal bovine serum, $100 \mathrm{U} / \mathrm{ml}$ penicillin, and $100 \mu \mathrm{g} / \mathrm{ml}$ streptomycin (Invitrogen Life Technologies, Carlsbad, CA, USA). The cells were maintained in an incubator at $37^{\circ} \mathrm{C}$ with an atmosphere of $5 \% \mathrm{CO}_{2}$, and were regularly checked for mycoplasma or other bacterial infections.

The study was approved by the Institutional Ethics Committee of the General Hospital of PLA (Beijing, China).

Immunohistochemical (IHC) staining. The KYSE170 and TE13 tumor-bearing mice were developed. At 2 weeks after cell injection, the tumors were surgically removed. The 5-mm tumor sections were fixed in formalin, mounted on a slide and deparaffinized with xylene, followed by treatment with an ethanol series. Citric acid was used to retrieve antigen at $90^{\circ} \mathrm{C}$ for $40 \mathrm{~min}$, following which the cells were treated with $3 \%$ hydrogen peroxide. The slides were incubated with rabbit polyclonal Ki-67, matrix metalloproteinase (MMP)-9, vascular endothelial growth factor (VEGF), cluster of differentiation (CD) 31 and IL-6 (1:1,000 dilution) antibodies at $4^{\circ} \mathrm{C}$. Antibodies were obtained from Biocare Medical (Concord, CA, USA), Santa Cruz Biotechnology, Inc. (Santa Cruz, CA, USA), Chemicon (Temecula, CA, USA), R\&D Systems, Inc. (Minneapolis, MN, USA) and R\&D Systems, Inc. The sections were washed three times with phosphate buffered saline (PBS) and incubated with a biotinylated secondary antibody for $10 \mathrm{~min}$. Next, the sections were stained with peroxidase-avidin, washed with PBS and treated with 3-amino-9-ethylcarbazole solution, prior to counter-staining with hematoxylin (all from Sigma-Aldrich, Shanghai, China). The IHC data were analyzed using the Image Pro Plus version 6.3 software (Media Cybernetics, Inc., Rockville, MD, USA). Subsequently, CD31 staining was used to evaluate the microvascular density (MVD) at x100 magnification. Staining of the specimens was assessed using a semi-quantitative immunoreactive score (IRS), which was determined by calculating the staining intensity [0, no staining $(<10 \%)$; 1 , weak staining $(11-50 \%)$; 2 , moderate staining $(51-80 \%)$; and 3 , strong staining $(\geq 81 \%)]$ based on the percentage of positively-stained cells. An IRS score of $>2$ was considered as positive staining.
Reverse transcription-quantitative polymerase chain reaction (RT-qPCR) experiment. The mRNA expression level IL-6 was investigated by means of RT-qPCR. Following overnight incubation at $4^{\circ} \mathrm{C}$ in $5 \%$ bovine serum albumin solution, total RNA from each sample was extracted using an RNeasy ${ }^{\circledR}$ Plus Mini kit (Qiagen, Hilden, Germany), according to the manufacturer's instructions. Total RNA $(2 \mu \mathrm{g})$ was reverse-transcribed using Super Script III (Invitrogen Life Technologies) and the following primers: Forward, 5'-TACATCCTCGACGGCATCTC-3' and reverse, 5'-GCTACATTTGCCGAAGAGCC-3' for IL-6. qPCR was performed with the 7900HT Real Time PCR System (Applied Biosystems, Carlsbad, CA, USA) using a Taqman ${ }^{\circledR}$ assay for IL-6 (Hs99999032_m1) (Invitrogen Life Technologies). PCR was performed under the following conditions: Initial denaturation at $95^{\circ} \mathrm{C}$ for $10 \mathrm{~min}$, followed by 40 cycles of $95^{\circ} \mathrm{C}$ for $15 \mathrm{sec}$, with a final extension step at $60^{\circ} \mathrm{C}$ for $1 \mathrm{~min}$. The GAPDH gene was used as the control.

Tumor xenograft model. All the animal protocols were approved by the Ethics Committee of the General Hospital of PLA (Beijing, China). Female athymic nude mice (weight, $20 \mathrm{~g}$; age, 8 weeks; n=48) obtained from the Institutional Animal Center of the General Hospital of PLA were used as xenograft tumor implantation models. The mice were caged in ambient conditions of $24^{\circ} \mathrm{C}$ with a relative humidity of $65 \%$ and an alternative 12-h light/dark cycle. In addition, the mice were provided with free access to food and water. KYSE170 or TE13 cells $\left(1 \times 10^{6}\right)$, transfected with IL-6 shRNA constructs were subcutaneously injected into the dorsal gluteal region. IL-6 expression was measured using a fluorescence microscope (FM200BA; AmScope, Irvine, CA, USA), as GFP expression vectors were used for the transfection. At 3 days after injection of the cells, the tumor volume was calculated, assuming an ellipsoidal shape. The effect of IL-6 expression on tumor growth was investigated by measuring tumor volume in vivo and constructing tumor growth curves. In the orthotopic tumor implantation model, intravesicular instillation of respective cancer cells was performed. The mice were sacrificed by $\mathrm{CO}_{2}$ asphyxiation at predetermined times $(3,6$, $9,12,15,18,21$ and 24 days) and the level of orthotopic tumor invasion was measured using a Vernier caliper (Thermo Fisher Scientific, Inc., Waltham, MA, USA). In addition, the effect of IL-6 stimulation was evaluated in vivo. For the four experimental groups (KYSE170, KYSE170-IL6 silencing vector, TE13, TE13-IL6 silencing vector; 12 mice per group), an intraperitoneal injection of IL-6 (75 ng/mouse, 3 times/week) was initiated 1 day prior to tumor implantation.

Cell migration and cell invasion assay. Cell invasion assays monitor cell movement through extracellular matrices. The cells $\left(5 \times 10^{5} /\right.$ well) were seeded on a 96 -well Boyden chamber. Cell invasion capacity was evaluated using a Cultrex ${ }^{\circledR}$ Laminin I Cell Invasion Assay (Trevigen ${ }^{\circledR}$, Gaithersburg, MD, USA). The upper chambers were coated with a membrane extract derived from Engelbreth-Holm-Swarm tumor. Following a 24-h incubation, the cell number in the bottom chamber was determined by the fluorescent anion calcein released from intracellular calcein acetoxymethyl ester. Calcein acetomethylester (AM) is internalized by the cells and intracellular esterases cleave 
A

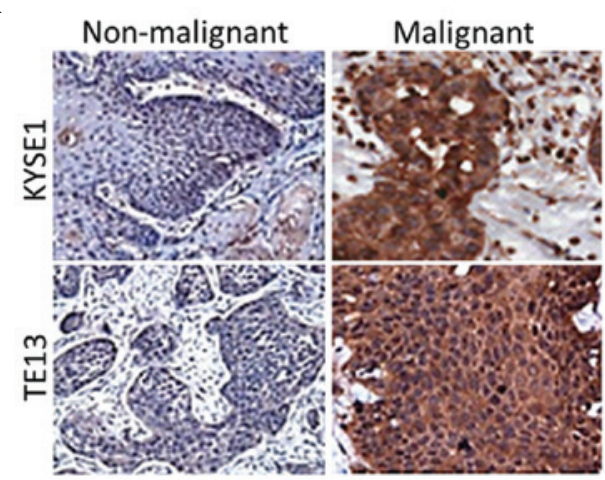

B

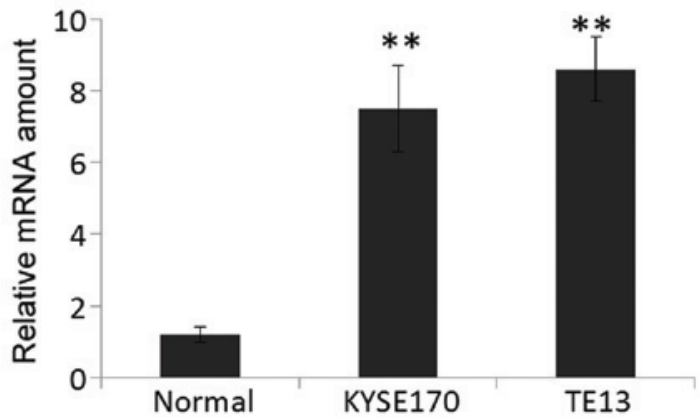

Figure 1. IL-6 expression in esophageal cancer cell lines injected into mouse models and in adjacent normal tissue. (A) Immunohistochemical staining of esophageal cancer specimens and adjacent non-malignant epithelium with anti-IL-6 antibody. (B) Levels of IL-6 were examined by reverse transcription-quantitative polymerase chain reaction. ${ }^{* *} \mathrm{P}<0.01$. IL-6, interleukin-6.

the AM moiety. Free Calcein fluoresces brightly and is used to quantitate the number of cells that have invaded in comparison to a standard curve. Thus, a SpectraMax Plus 384 microplate reader (Molecular Devices, LLC, Sunnyvale, CA, USA) was used to measure the fluorescence at $485 \mathrm{~nm}$ for excitation and $520 \mathrm{~nm}$ for emission. Images were captured using a confocal laser scanning microscope (TE2000-E; Nikon Corporation, Tokyo, Japan). To further assess cell migration, scratch assays were conducted to measure the number of cells traversing a porous membrane. KYSE170 and TE13 cells were cultured to $\sim 80 \%$ confluence. Twenty four hours prior to the experiment, cells were resuspended at $1 \times 10^{6} \mathrm{cell} / \mathrm{ml}$ basal medium containing DMEM with 5\% stripped serum (Gibco; Thermo Fisher Scientific, Inc., Waltham, MA, USA). To prevent proliferation, on day 0 cells were treated with $8 \mu \mathrm{g} / \mathrm{ml}$ mitomycin $\mathrm{C}$ (ICN Biomedicals, Costa Mesa, CA, USA) for $1 \mathrm{~h}$, and washed with PBS. A pipette tip was used to draw a $2 \mathrm{~mm}$-wide scratch across each cell layer and images were captured using a Carl Zeiss microscope (Axio Vert.A1; Carl Zeiss AG, Oberkochen, Germany) and a digital camera (DKC-500; Sony Corporation, Tokyo, Japan). Cells were then incubated for $24 \mathrm{~h}$ and images were recaptured in the same fields.

Western blotting. Cells were lysed by treating with CelLytic ${ }^{\mathrm{TM}}$ MT Cell Lysis Reagent (Sigma-Aldrich). An NE-PER ${ }^{\mathrm{TM}}$ kit (Pierce Biotechnology, Inc., Rockford, IL, USA) was used to isolate nuclear and cytoplasmic proteins. An equimolar quantity of protein was then subjected to SDS-PAGE, following which proteins were transferred onto nitrocellulose filters. The blots were blocked with $2 \%$ bovine serum albumin solution for $1 \mathrm{~h}$ and subsequently incubated overnight at $4^{\circ} \mathrm{C}$ with polyclonal goat anti-mouse IL-6 (cat. no. sc-1265; Santa Cruz Biotechnology, Inc.), rabbit anti-mouse IL-6 receptor (IL-6R; cat. no. sc-660; Santa Cruz Biotechnology, Inc.), mouse anti-human VEGF (cat. no. MAB293; R\&D Systems, Inc.), rat anti-mouse E-cadherin (cat. no. 748-EC; R\&D Systems, Inc.), anti-human mouse STAT3 (cat. no. MAB1799; R\&D Systems, Inc.) and polyclonal goat anti-human MMP-9 (cat. no. AF911; R\&D Systems, Inc.) (1:1,200 dilution) antibodies. The membranes were further incubated with horseradish peroxidase-conjugated secondary rabbit anti-goat (cat. no. ab6741), rabbit anti-mouse (cat. no. ab6728) or goat anti-rabbit (cat. no. ab6721) IgG antibodies (dilution, 1:1,000-1:2,000; Abcam, Cambridge, MA, USA), for $1 \mathrm{~h}$ at room temperature. The film was developed using an X-ray processor and visually examined to determine the intensity of the protein band.

Immunofluorescence staining. Cells $\left(5 \times 10^{6}\right)$ were seeded onto a cover slip with and without respective treatments. After $24 \mathrm{~h}$ incubation, cells were fixed, permeabilizeda with $4 \%$ paraformaldehyde and washed with PBS containing Tween 80 . The slides were then incubated with antibodies against E-cadherin, IL-6 and p-STAT3 for $1 \mathrm{~h}$, followed by incubation with Texas Red-conjugated secondary antibody. The slides were counter stained with DAPI, washed and visualized using a Meiji Techno MT6000 Epi-Fluorescence microscope (The Microscope Store, LLC, Roanoke, VA, USA).

Enzyme-linked immunosorbent assay (ELISA) of IL-6 levels in vitro and in vivo. IL-6 levels in KYSE170 and TE13 cell supernatant and serum samples from mice injected with cancer cells were analyzed using an IL-6 Quantikine ELISA kit (R\&D Systems, Inc.). IL-6 levels in cell supernatants were measured by culturing the cells $\left(5 \times 10^{5}\right)$ with $1 \mathrm{ml}$ serum-free media in 6-well plates and incubating for $24 \mathrm{~h}$ at $37^{\circ} \mathrm{C}$. Next, the media was centrifuged at $800 \mathrm{x}$ g for $5 \mathrm{~min}$ and collected. Similarly, IL-6 levels in in vivo conditions were evaluated by removing or separating blood from the serum by centrifugation at $5000 \mathrm{x} \mathrm{g}$ for $15 \mathrm{~min}$ and performing an ELISA.

Statistical analysis. Student's t-test was employed to analyze the results. Data are presented as the mean \pm standard error of the mean, and experiments were performed in triplicate. $\mathrm{P}<0.05$ was considered to indicate a statistically significant difference, unless otherwise stated.

\section{Results}

Expression of IL-6 in esophageal SCC. Identifying the key molecules and mechanisms of esophageal cancer growth is essential. A novel therapeutic target that is able to affect cancer cell metastasis and proliferation will aid in improving the prognosis and suppressing the progression of the disease (18). It has been previously reported that elevated levels of IL- 6 in the blood 
$\mathbf{A}$ KYSE170

IL-6

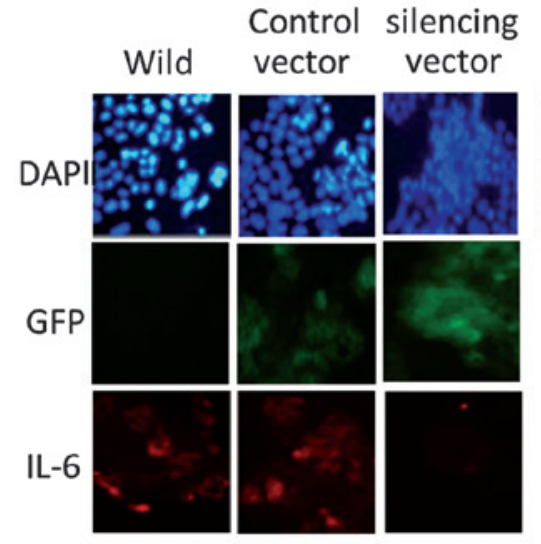

C

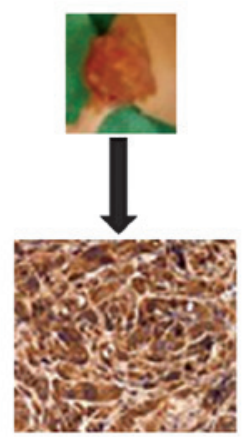

TE13

IL-6 Control silencing Wild
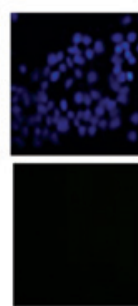

vector vector
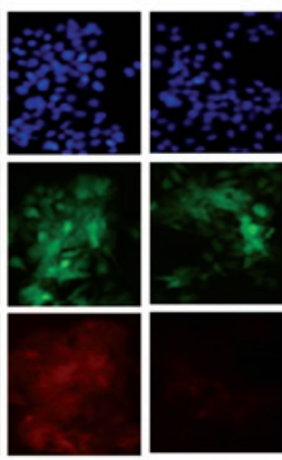

B

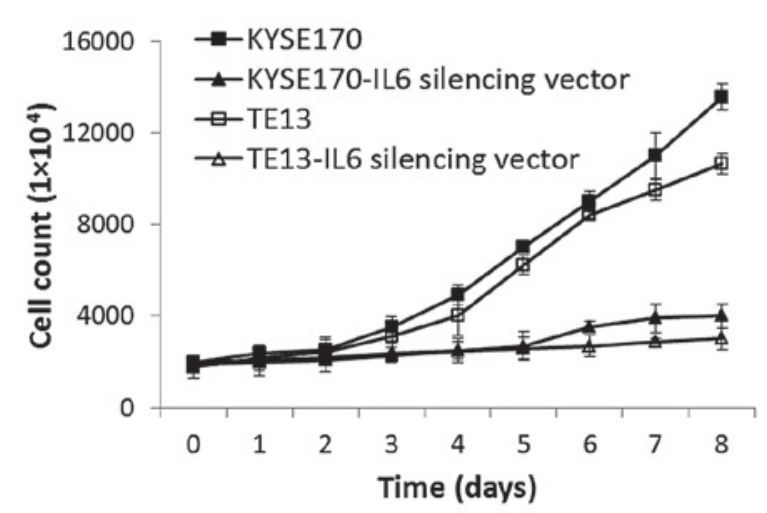

D

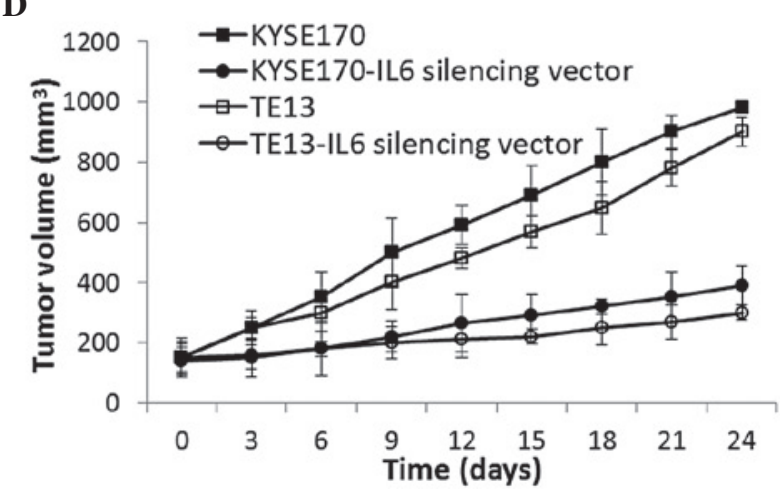

Figure 2. Effect of IL-6 on tumor cell growth. (A) Immunofluorescence analysis of IL-6 levels in KYSE170 and TE13 cells transfected with IL-6-GFP silencing vector and untransfected (wild) cells. (B) Effect of IL-6 on the proliferation rates of KYSE170 and TE13 cancer cell lines as determined by viable cell counting. (C) IL-6 expression in tumors evaluated by immunohistochemical staining of xenografts. (D) Effects of IL-6 inhibition on the relative tumor volume of experimental groups. IL-6, interleukin 6; GFP, green fluorescent protein.

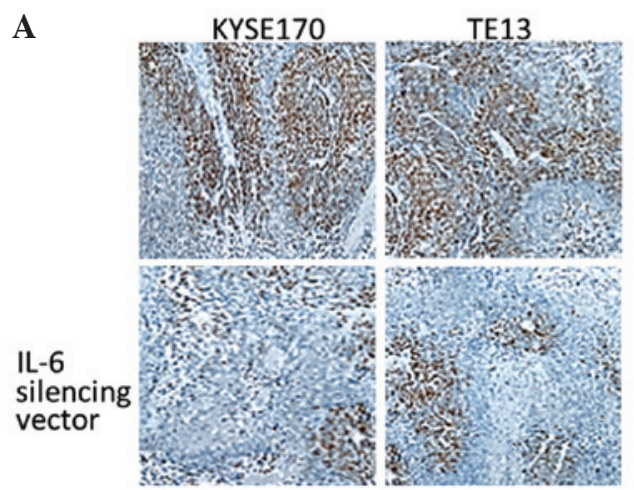

C

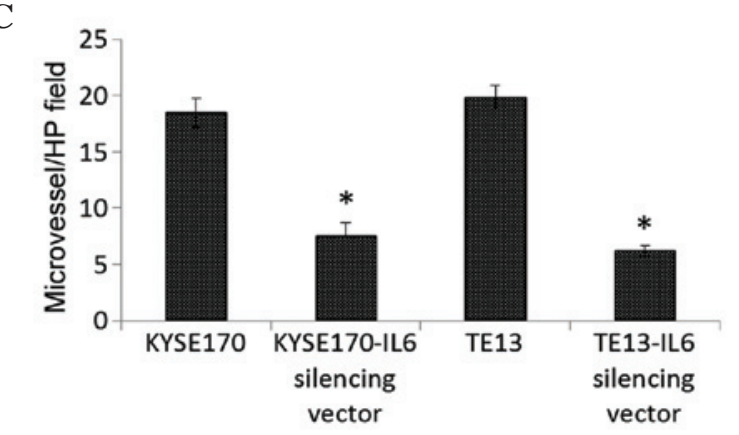

B

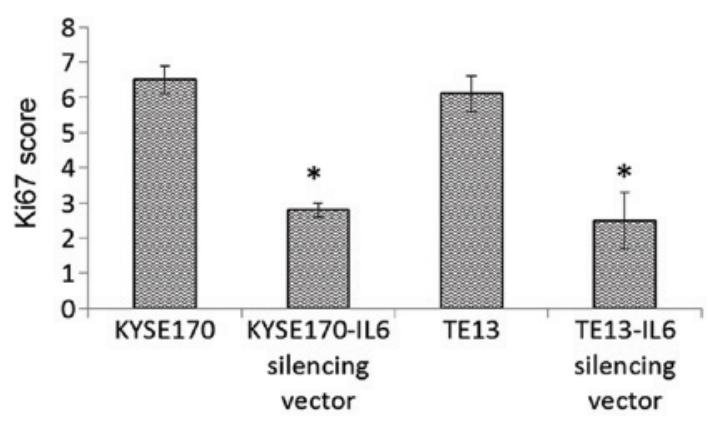

Figure 3. Downregulation of IL-6 in tumor-associated endothelial cells reduces the tumor proliferation. (A) Immunohistochemical analysis of Ki-67 expression, representing tumor cell proliferation, in cancer cells following IL-6-silencing (magnification, x100). (B) Quantification of tumor cell proliferation determined by scoring Ki-67 immunostaining. (C) Quantification of tumor cell proliferation determined by microvessel density. "P<0.05. IL-6, interleukin 6; HP, high power. 
A
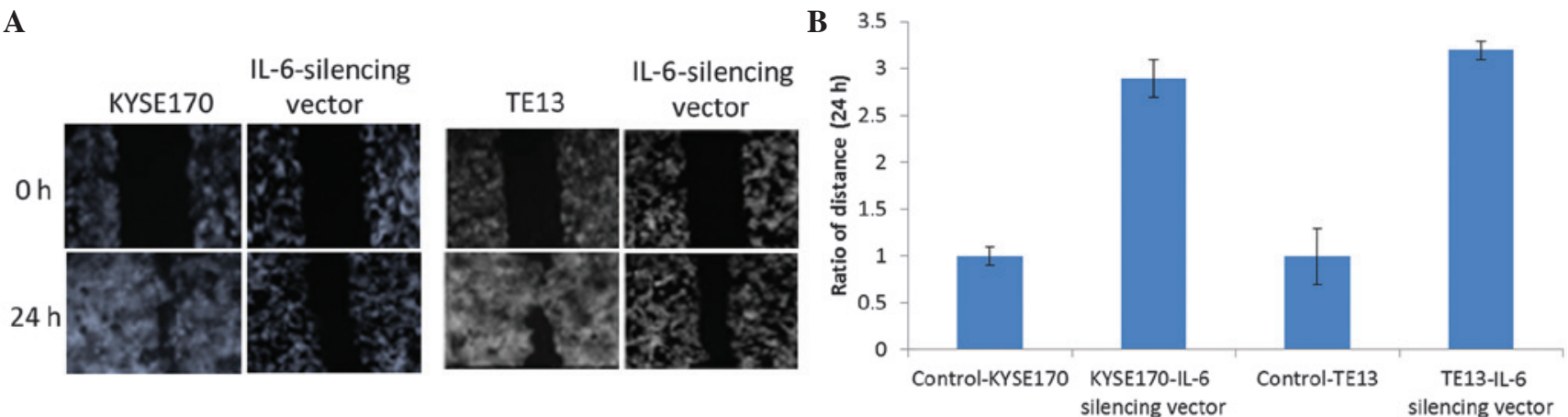

C

KYSE170

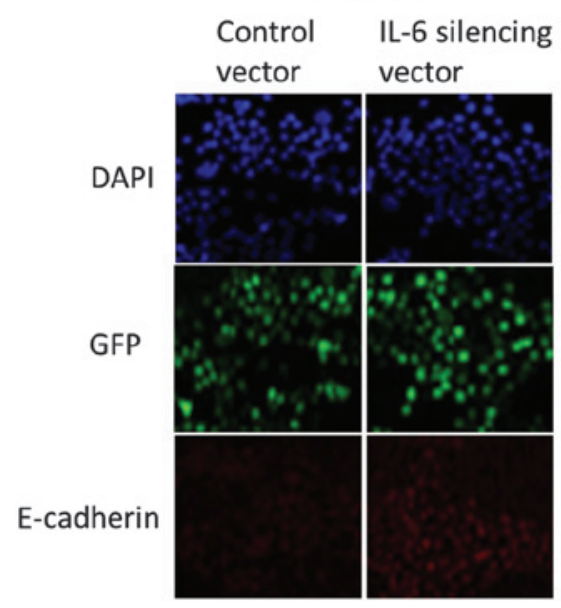

TE13

D
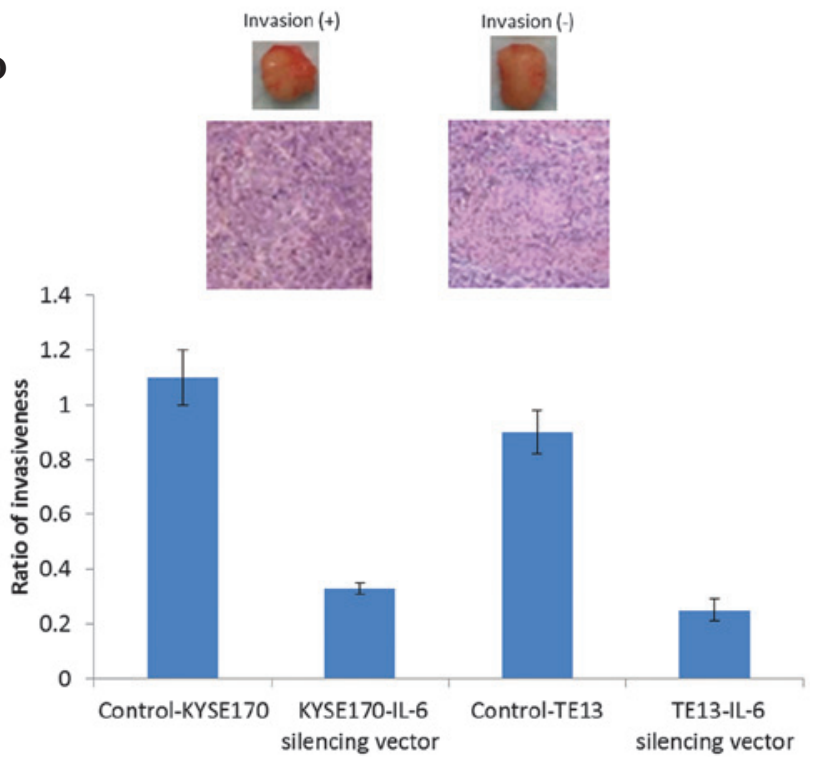

Figure 4. Effect of IL-6 inhibition on aggressive tumor behavior and epithelial-mesenchymal transition changes. (A) Effect of IL-6 inhibition on the migration/invasive ability of esophageal cancer cells (KYSE170 and TE13), as measured by a scratch assay. Images of the plates were captured at the times indicated. (B) The migration ability of esophageal cancer cells with or without the IL-6 silencing vector was evaluated by murine orthotopic tumor implantation. (C) E-cadherin levels in cells were evaluated by immunofluorescence assay. The immunofluorescent colors are as follows: Blue, DAPI; green, GFP; red, E-cadherin. (D) The invasive capacity of cancer cells as determined by cell invasion assay; representative slides and quantitative data are shown. Slides represent KYSE170-IL-6 silencing vector-treated mice (right) and TE13-IL-6 silencing vector-treated mice (left). IL-6, interleukin 6; GFP, green fluorescent protein.

are associated with poor prognosis and disease progression. By activating JAK/STAT3 and PI3K/AKT signalling pathways, IL-6 is able to regulate the growth and spread of cancer in the body $(17,19,20)$. However, at present, little information is available regarding the role of IL-6 in esophageal cancer.

KYSE170 and TE13 tumor models were developed by injecting the respective cell lines into xenograft mice. The tumor was removed and IHC analysis was performed on specimens of tumor tissue and the adjacent non-malignant epithelium (Fig. 1A), along with quantification of IL-6 mRNA expression (Fig. 1B). The IHC analysis demonstrated positive staining for IL-6 in specimens injected with the two cell lines. Specifically, $70-80 \%$ of cancer cells exhibited positive staining for IL-6, compared with $<15 \%$ for non-malignant epithelial cells. RT-qPCR was performed to quantify the mRNA expression of these tissue specimens. Consistent with the IHC analysis, cancerous cells expressed significantly higher levels of IL-6 mRNA compared with normal epithelia $(\mathrm{P}=0.0021)$.

Effect of IL-6 expression on tumor growth. KYSE170 and TE13 cells were transfected with IL-6-GFP silencing vector to determine the role of IL-6 in the growth of esophageal carcinoma. As shown in Fig. 2A, the IL-6-GFP silencing vector markedly decreased the expression of IL-6 in the two cell lines, as demonstrated by a clear reduction in the intensity of red fluorescence (indicating IL-6 expression) compared with that of control-vector-transfected cells. This was accompanied by differences in the viable cell count over 8 days: The IL-6 silencing vector significantly reduced the proliferation of the two cell lines compared with the control-vector-transfected cells throughout the study period ( $\mathrm{P}=0.0013$; Fig. 2B). In addition, the silencing vector significantly attenuated the growth of tumors in the xenograft mouse models $(\mathrm{P}=0.0031)$. The tumor volume in mice bearing KYSE170 and TE13 tumors increased significantly more rapidly (up to $\sim 1,000 \mathrm{~mm}^{3}$ ) $(\mathrm{P}=0.002)$, while the IL-6 vector was found to inhibit growth by $\sim 300 \mathrm{~mm}^{3}$ by controlling the vascular endothelial cells (Fig. 2C and D). Furthermore, marked inhibition of tumor invasion was observed in the IL-6 silencing vector group in the two tumor models. Therefore, based on the cell and tumor growth analysis, it is hypothesized that IL-6 has a direct or indirect role in angiogenesis and esophageal cancer 
A

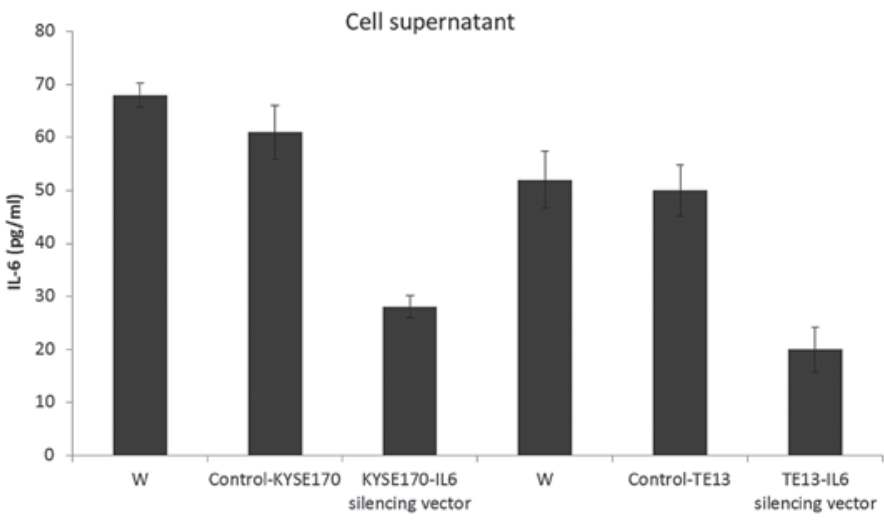

B

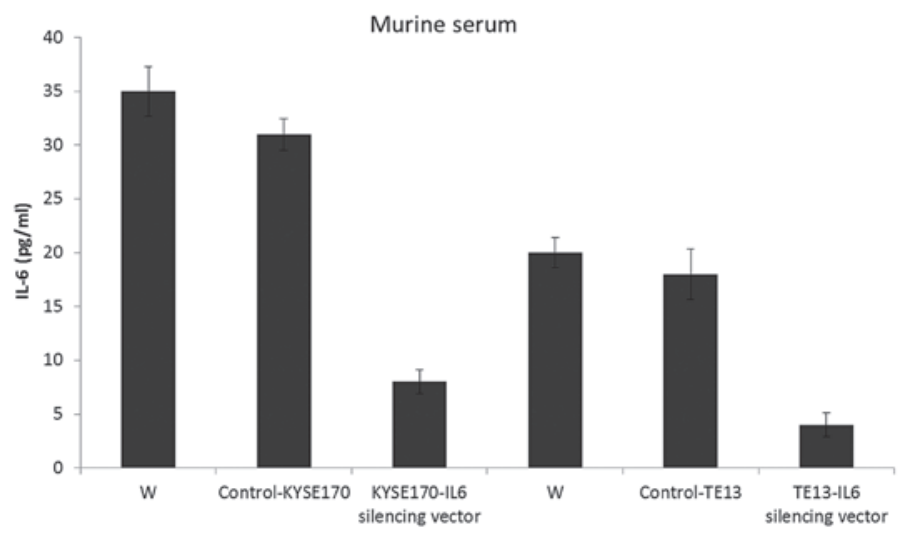

Figure 5. Effects of circulating IL-6 on tumor aggressiveness. Enzyme-linked immunosorbent assay was used to evaluate the IL-6 level produced from KYSE170 and TE13 cells with or without the IL-6 silencing vector in (A) cell culture supernatants and (B) the serum of mice bearing KYSE170 and TE13 tumors. IL-6, interleukin 6; W, untreated mice.

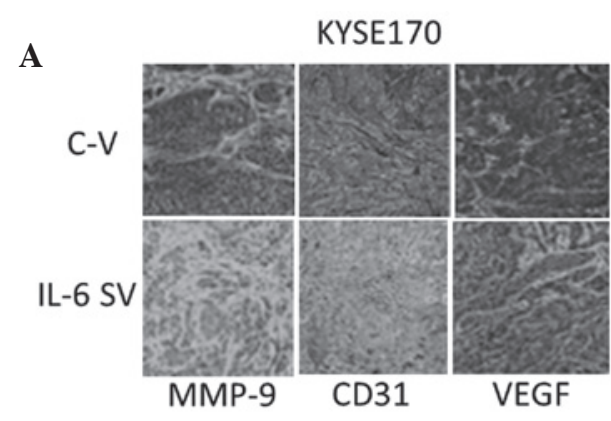

$\mathbf{C}$

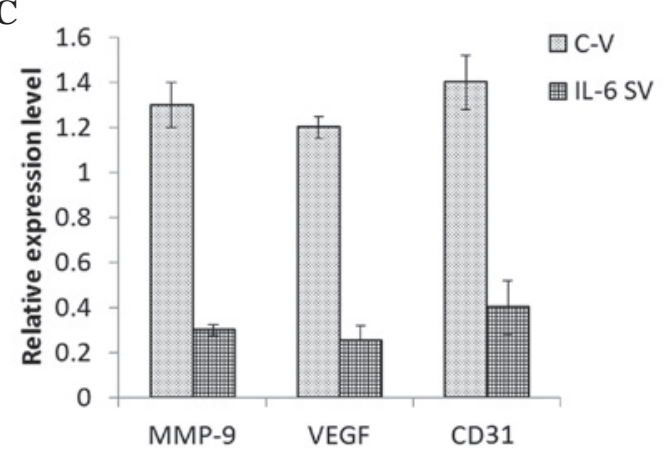

B

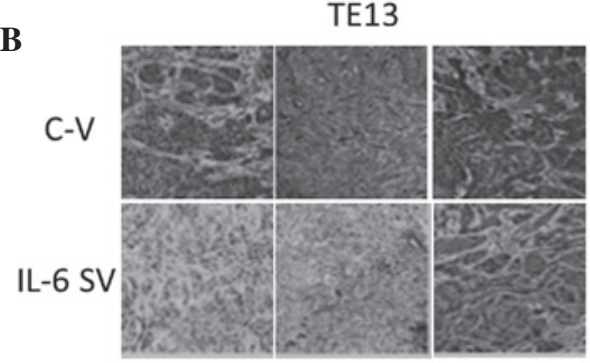

D

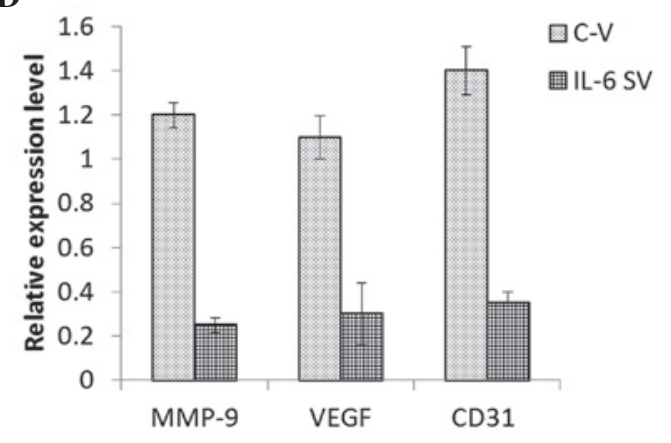

Figure 6. Effects of circulating IL-6 on tumor aggressiveness. Immunohistochemical staining of MMP-9, CD31, and VEGF in tumors (A) 24 days after KYSE170 tumor cell implantation and (B) 24 days after TE13 tumor cell implantation. (C and D) Quantitative estimation of arbitrary values determined from (A) and (B), respectively. MMP-9, matrix metalloproteinase 9; CD31, cluster of differentiation 31; VEGF, vascular endothelial growth factor; C-V, control vector; $\mathrm{SV}$, silencing vector.

progression. Angiogenesis is one of the key processes involved in the proliferation, progression and secretion of growth factors of tumor cells that will have great significance in the final outcome of patients $(21,22)$. 
IL-6 in tumor-associated endothelial cells. Since the tumor inhibition properties of the IL-6 silencing vector were demonstrated, the underlying mechanism of tumor suppression was the investigated by assessing tumor cell proliferation and MVD through immunohistochemical assay. The IL-6 silencing vector significantly reduced the expression of $\mathrm{Ki}-67$, a typical proliferation marker, in the two esophageal cancer cell lines (Fig. 3A and B). MVD was also significantly reduced in the vector-treated group compared with that of the non-silenced group (control endothelial cells) $(\mathrm{P}=0.0023)$ indicating that downregulation of IL-6 level may have a marked effect on the tumor growth and inhibition (Fig. 3C).

A low Ki-67 expression and MVD in tumor cells are positive signs towards the development of a therapeutic target based on IL-6. It has been previously reported that B-cell lymphoma-2 is upregulated in tumor-associated endothelial cells and that its regulation accelerates tumor growth (23). Therefore, a better understanding of the biological mechanisms/therapeutic targets involved in the progression and development of respective cancers may aid in the improvement of pharmacological interventions.

IL-6 in tumor invasion and epithelial-mesenchymal transition (EMT)-associated changes. Migration scratch assays clearly demonstrated the ability of the IL-6 silencing vector to inhibit the invasion capacity of the two esophageal cancer cell lines. As shown in Fig. 4A, the normal endothelial cells invade from both sides, whereas presence of the silencing vector effectively prevented this invasion. Fig. 4B shows the quantitative presentation of the aforementioned results. Furthermore, the EMT is a key process in invasiveness. As shown in Fig. 4C, the IL-6 silencing vector increased the expression of the E-cadherin in the two esophageal carcinoma cell lines. Similarly, it may be expected that MMP-9 levels decrease with E-cadherin levels (24).

Additionally, an orthotopic tumor implantation technique was employed to evaluate the tumor invasive properties in vivo. For this purpose, 20 mice were selected and injected with esophageal cell lines intravesicularly. Subsequently, the tumors developed in each of the 4 groups (KYSE; KYSE+IL-6 vector; TE13; and TE13+IL-6 vector) were evaluated. As shown in Fig. 4D, compared with control endothelial groups (KYSE and TE13 cells), the IL-6 vector-treated group exhibited significantly reduced tumor invasion in vivo $(\mathrm{P}=0.016)$.

Effects of circulating IL-6 on angiogenesis. An ELISA was employed to quantify the IL-6 levels in the supernatant and serum of mice in the four treatment groups. As shown in Fig. 5, the IL-6 silencing vector significantly regulated the release of IL-6 in the cell supernatant compared with that of controls in KYSE170 tumor-bearing mice $(\mathrm{P}=0.019)$. Similar results were observed in TE13 tumor-bearing mice. In the same line, the IL-6 silencing vector markedly controlled the release of IL-6 in the serum of the mice, which was collected 24 days after the implantation or injection of cancer cells. Furthermore, angiogenesis in tumors excised from the experimental animals was evaluated by staining for CD31 and VEGF, which are prominent members of the angiogenic pathways essential for tumor growth (25), and are involved in the angiogenic pathways throughout tumor cell proliferation. Therefore, vascular networks in the tumors were stained for VEGF and CD31 (26). The tumors were then surgically removed and immunohistochemically analyzed. The IL-6 silencing vector markedly reduced the secretion of VEGF and CD31 (a nuclear protein) compared with that of control tumors, which showed dark staining for these immunoreactive molecules (Fig. 6). Therefore, IL-6 may be a major trigger for the progression of angiogenesis and endothelial tube formation within the tumor.

\section{Discussion}

The cytokine IL-6 is a key inflammatory molecule involved in the generation and proliferation of various types of cancer, including gastrointestinal and prostate cancer, and also functions as an autocrine growth factor (27). Furthermore, IL-6 plays a crucial role as an antiapoptotic factor, such as in the IL-6/STAT3 pathway, which may lead to further inflammation and growth of cancer cells (28). Recently, a number of studies have been published regarding the role of IL-6 in esophageal cancer. Cancer cells positively stained for IL-6 were likely to be in advanced stages of tumor development (29). Therefore, in the present study, IL-6 expression or release was suppressed with IL-6 silencing vector in two types of esophageal cancer. IL-6 was demonstrated to be a major cue for angiogenesis, which in turn facilitates tumor growth.

The current results demonstrated that IL-6 was accumulated in markedly higher levels in esophageal cancer cells compared with the levels in the normal epithelium. By inhibiting IL-6, tumor growth was found to be significantly inhibited in vitro and in vivo. Silencing of IL-6 also resulted in the significant attenuation of tumor growth and invasive ability in specific cell lines $(\mathrm{P}<0.05)$. This is an important observation as the transformation of epithelial cells into mesenchymal cells depends on the invasive character of the tumor cells (30).

A previous study has reported that STAT3 plays an important role in tumor progression and behavior and, thereby, EMT changes in the cells $(31,32)$. In addition, it was demonstrated that IL- 6 can activate the JAK/STAT3 signaling pathways and is actively involved in tumor proliferation and prognosis (33). Furthermore, STAT3 activation was reported to be involved in the CIS progression pathway in esophageal cancer. Therefore, it can be expected that IL- 6 plays a central role in the regulation of EMT changes and invasiveness.

The results also demonstrated a decreased level of IL-6 in the supernatant of cell culture medium, as well as in the serum collected from the tumor-bearing mice. Angiogenesis plays a vital role in tumor cell proliferation and progression (34). The present study observed that CD31 and VEGF mediated endothelial cell-cell interactions and actively participated in angiogenesis. Furthermore, IL-6 was observed to play a crucial role in the in vivo tumor growth and tumor progression. These observations indicate that EMT-associated changes and angiogenesis are interrelated and involved in aggressive tumor growth in esophageal cancer. Further results suggested that IL- 6 provides a suitable in vivo environment for the growth and development of tumor cells. Previous studies have reported that it may lead to resistance to certain anticancer drugs by promoting angiogenesis and cell mobility (35). Mian et al (36) reported that 
inhibition of IL- 8 markedly suppressed the expression levels of MMP-2 and MMP-9 in cancer cells, leading to a decrease in invasion and an increase in the progression of disease symptoms. Therefore, the in vivo biological and physiological mechanisms linking the various cytokines, including IL-6, IL-8 and TNF- $\alpha$, are difficult to establish, and further mechanistic study is warranted in this regard. Overall, the experimental results of the present study indicate that IL-6 is a crucial element involved in tumor growth. Furthermore, IL-6 is a potentially significant biological marker and may represent a promising therapeutic target in the treatment of esophageal carcinomas.

In conclusion, the current study demonstrated that IL-6 is responsible for inflammatory cytokine production, tumor angiogenesis and tumor growth in esophageal cancer cell lines (KYSE170 and TE13), and these functions may be effectively countered by inhibiting IL-6 production using an IL-6 silencing vector. Based on these results, IL- 6 may be used as a potential therapeutic target in the treatment of esophageal cancer. Future studies should focus on further investigating the inhibitory effect of anticancer drugs on the molecular mechanisms of IL-6.

\section{Acknowledgements}

The authors would like to thank all those who were involved in conducting the study, and Dr Wei Lin (Southern Medical University, Guangzhou, China) and Dr Ziang Chen (The Rirst Affiliated Hospital of Shenzhen University, China) for proofreading the manuscript.

\section{References}

1. Jemal A, Bray F, Center MM, Ferlay J, Ward E and Forman D: Global cancer statistics. CA Cancer J Clin 61: 69-90, 2011.

2. Shahbaz Sarwar CM, Luketich JD, Landreneau RJ and Abbas G: Esophageal cancer: An update. Int J Surg 8: 417-422, 2010.

3. Jemal A, Siegel R, Ward E, Hao Y, Xu J and Thun MJ: Cancer statistics, 2009. CA Cancer J Clin 59: 225-249, 2009.

4. Allum WH, Stenning SP, Bancewicz J, Clark PI and Langley RE: Long-term results of a randomized trial of surgery with or without preoperative chemotherapy in esophageal cancer. J Clin Oncol 27: 5062-5067, 2009.

5. Sjoquist KM, Burmeister BH, Smithers BM, Zalcberg JR, Simes RJ, Barbour A and Gebski V; Australasian Gastro-Intestinal Trials Group: Survival after neoadjuvant chemotherapy or chemoradiotherapy for resectable oesophageal carcinoma: An updated meta-analysis. Lancet Oncol 12: 681-692, 2011.

6. Chen WQ, Zheng RS, Zhang SW, Li N, Zhao P, Li GL, Wu LY and He J: Report of incidence and mortality in china cancer registries, 2008. Chin J Cancer Res 24: 171-180, 2012.

7. Liu Q, Li R, Zhu Z, Qian X, Guan W, Yu L, Yang M, Jiang X and Liu B: Enhanced antitumor efficacy, biodistribution and penetration of docetaxel-loaded biodegradable nanoparticles. Int J Pharm 430: 350-358, 2012.

8. Coussens LM and Werb Z: Inflammation and cancer. Nature 420: 860-867, 2002.

9. Balkwill $\mathrm{F}$ and Mantovani A: Inflammation and cancer: Back to Virchow? Lancet 357: 539-545, 2001.

10. Gonda TA, Tu S and Wang TC: Chronic inflammation, the tumor microenvironment and carcinogenesis. Cell Cycle 8: 2005-2013, 2009.

11. Abdel-Latif MM, Duggan S, Reynolds JV and Kelleher D: Inflammation and esophageal carcinogenesis. Curr Opin Pharmacol 9: 396-404, 2009.

12. Schafer ZT and Brugge JS: IL-6 involvement in epithelial cancers. J Clin Invest 117: 3660-3663, 2007.

13. Akira $\mathrm{S}$, Taga $\mathrm{T}$ and Kishimoto T: Interleukin-6 in biology and medicine. Adv Immunol 54: 1-78, 1993.
14. Chen CC, Chen WC, Lu CH, Wang WH, Lin PY, Lee KD and Chen MF: Significance of interleukin-6 signaling in the resistance of pharyngeal cancer to irradiation and the epidermal growth factor receptor inhibitor. Int J Radiat Oncol Biol Phys 76: 1214-1224, 2010.

15. Santer FR, Malinowska K, Culig Z and Cavarretta IT: Interleukin-6 trans-signalling differentially regulates proliferation, migration, adhesion and maspin expression in human prostate cancer cells. Endocr Relat Cancer 17: 241-253, 2010.

16. Kishimoto T: Interleukin-6: From basic science to medicine - 40 years in immunology. Annu Rev Immunol 23: 1-21, 2005.

17. Guo Y, Xu F, Lu T, Duan Z and Zhang Z: Interleukin-6 signaling pathway in targeted therapy for cancer. Cancer Treat Rev 38: 904-910, 2012.

18. Stahl M: Is there any role for surgery in the multidisciplinary treatment of esophageal cancer? Ann Oncol 21 (Suppl 7): vii283-vii285, 2010.

19. Trikha M, Corringham R, Klein B and Rossi JF: Targeted anti-interleukin-6 monoclonal antibody therapy for cancer: A review of the rationale and clinical evidence. Clin Cancer Res 9: 4653-4665, 2003.

20. Leu CM, Wong FH, Chang C, Huang SF and Hu CP: Interleukin-6 acts as an antiapoptotic factor in human esophageal carcinoma cells through the activation of both STAT3 and mitogen-activated protein kinase pathways. Oncogene 22: 7809-7818, 2003.

21. Chen MF, Lee KD, Lu MS, Chen CC, Hsieh MJ, Liu YH, Lin PY and Chen WC: The predictive role of E2-EPF ubiquitin carrier protein in esophageal squamous cell carcinoma. J Mol Med (Berl) 87: 307-320, 2009.

22. Thiery JP: Epithelial-mesenchymal transitions in tumour progression. Nat Rev Cancer 2: 442-454, 2002.

23. Scott DW and Gascoyne RD: The tumour microenvironment in B cell lymphomas. Nat Rev Cancer 14, 517-534, 2014.

24. McConkey DJ, Choi W, Marquis L, Martin F, Williams MB, Shah J, Svatek R, Das A, Adam L, Kamat A, et al: Role of epithelial-to-mesenchymal transition (EMT) in drug sensitivity and metastasis in bladder cancer. Cancer Metastasis Rev 28: 335-344, 2009.

25. Tartour E, Pere H, Maillere B, Terme M, Merillon N, Taieb J, Sandoval F, Quintin-Colonna F, Lacerda K, Karadimou A, et al: Angiogenesis and immunity: A bidirectional link potentially relevant for the monitoring of antiangiogenic therapy and the development of novel therapeutic combination with immunotherapy. Cancer Metastasis Rev 30: 83-95, 2011.

26. Sharma S, Sharma MC and Sarkar C: Morphology of angiogenesis in human cancer: A conceptual overview, histoprognostic perspective and significance of neoangiogenesis. Histopathology 46: 481-489, 2005.

27. Landskron G, De la Fuente M, Thuwajit C and Hermoso MA: Chronic inflammation and cytokines in the tumor microenvironment. J Immunol Res 2014, 2014.

28. Sepúlveda P, Encabo A, Carbonell-Uberos F and Miñana MD: BCL-2 expression is mainly regulated by JAK/STAT3 pathway in human CD34+ hematopoietic cells. Cell Death Differ 14: 378-380, 2007.

29. Aggarwal BBA, Kunnumakkara AB, Harikumar KB, Gupta SR, Tharakan ST, Koca C, Dey S and Sung B: Signal transducer and activator of transcription-3, inflammation, and cancer: How intimate is the relationship? Ann N Y Acad Sci 1171: 59-76, 2009.

30. Levy DE and Darnell JE Jr: Stats: Transcriptional control and biological impact. Nat Rev Mol Cell Biol 3: 651-662, 2002.

31. Bromberg J: Stat proteins and oncogenesis. J Clin Invest 109: 1139-1142, 2002

32. Bishop JL, Thaper D and Zoubeidi A: The multifaceted roles of STAT3 signaling in the progression of prostate cancer. Cancers (Basel) 6: 829-859, 2014.

33. Chen MF, Lu MS, Lin PY, Chen PT, Chen WC and Lee KD: The role of DNA methyltransferase 3b in esophageal squamous cell carcinoma. Cancer 118: 4074-4089, 2012.

34. Sarvaiya PJ, Guo D, Ulasov I, Gabikian P and Lesniak MS: Chemokines in tumor progression and metastasis. Oncotarget 4: 2171-2185, 2013.

35. Schindler C, Levy DE and Decker T: JAK-STAT signaling: From interferons to cytokines. J Biol Chem 282: 20059-20063, 2007.

36. Mian BM, Dinney CP, Bermejo CE, Sweeney P, Tellez C, Yang XD, Gudas JM, McConkey DJ and Bar-Eli M: Fully human anti-interleukin 8 antibody inhibits tumor growth in orthotopic bladder cancer xenografts via down-regulation of matrix metalloproteases and nuclear factor-kappaB. Clin Cancer Res 9: 3167-3175, 2003. 